Aim To explore the palliative care needs of adult patients and their informal carers living with MND, or bereaved carers of people with $\mathrm{MND}$, through a systematic review of qualitative research.

Method Searches were conducted in four electronic databases (MEDLINE, CINAHL, PsycINFO, Social Science Citation Index) using terms for MND, ALS, palliative care, and a qualitative research filter. Relevant data were extracted from included papers, which were also appraised for quality. The review was conducted using Thematic Synthesis, with the papers coded in order of disease trajectory.

Results 410 papers were identified and 39 included, representing the experiences of 456 people with MND and 345 informal caregivers.

Two sets of analytical themes were identified. The first represents the disease trajectory and specific chronological points within it. The second details three sets of factors (internal, external and communicating) that impact on people's experiences of events along the disease trajectory. Analysing the intersections of these themes highlighted which palliative care needs are most required at each stage.

The findings highlighted the need for greater communication and information provision to allow timely, informed decision making, empowering individuals to maintain control of their care in face of the unremitting loss caused by the disease.

Conclusion Despite being a life-limiting condition people with MND and their carers have limited awareness of, or involvement with, palliative care services. There are clear points in the disease trajectory where palliative care input could enhance patient and carer experience of the disease, particularly at times of significant physical change.

\section{P-99 MULTI-DISCIPLINARY PALLIATIVE CARE FOR MEN LIVING WITH DUCHENNE: A QUALITATIVE INTERVIEW STUDY}

Sheonad Laidlaw, Emma Carduff. Marie Curie Care, Glasgow, UK

\subsection{6/bmjspcare-2018-hospiceabs. 124}

Background Duchenne Muscular Dystrophy (DMD) is an $\mathrm{x}$ linked, ultra-rare neuromuscular condition affecting 1 in 3600-6000 live male births (Bushby, Finkel, Birnkrant et al., 2010). Individuals live with an exceptional illness trajectory of prolonged dwindling, frailty and high symptom burden (Landfeldt, Lindgren, Bell et al., 2014). While it is recognised that a co-ordinated multidisciplinary team approach may increase the survival of those with DMD and improve their quality of life (QoL) (Bushby, Finkel, Birnkrant et al., 2010), adults are receiving less comprehensive and co-ordinated care compared with those in the paediatric service (Rodger, Woods, Bladen et al., 2015).

Aim To investigate QoL in adults with DMD living in the West of Scotland (WoS).

Methods The SEIQoL-DW tool was used to assess the five most important elements that contribute to an individual's QoL - these were then used to guide qualitative interviews with six men in the WoS. A thematic analysis was undertaken. Results Men living with DMD in the WoS described living good lives, but feel 'forgotten' due to perceived gaps in their care: poorly co-ordinated and infrequent health care; lack of multi-disciplinary team input and holistic care; and poor or no access to allied health care professionals, for example physiotherapy and psychological support.

Conclusions Existing guidelines rarely seem to materialise as person-centred care. There are numerous opportunities to introduce palliative care gently, as part of the MDT, early in the illness trajectory and continue in a dynamic manner as time elapses and when trigger points arise. Better co-ordinated multi-disciplinary care, with the inclusion of a palliative care specialist, may be a solution allowing for an early introduction to palliative care and proactive advance care planning.

\section{P-100 DELIVERING COLLABORATIVE EDUCATION TO ENHANCE PATIENT-CENTRED DECISION MAKING IN RENAL FAILURE}

'Sue Goodall, ${ }^{2}$ Pamela Dallyn, 'Suzanne Ford-Dunn. 'St Barnabas House Hospice, Worthing, UK; ${ }^{2}$ Sussex Kidney Unit, Brighton, UK

10.1136/bmjspcare-2018-hospiceabs. 125

Background There is increasing recognition that dialysis may not offer significant survival advantage or improvement of symptoms, in older patients with high co-morbidity (Hussain, Mooney \& Russon, 2013; Chandna, Da Silva-Gane, Marshall et al., 2011; Davidson \& Sheerin, 2013; Verberne, Geers, Jellema et al., 2016; Foote, Kotwal, Gallagher et al., 2016). Despite this the number of people over the age of 75 starting dialysis in the UK continues to increase (UK Renal Registry, 2017). Several studies have raised concern that patients are not fully informed about conservative management, or the risks and burdens associated with dialysis, (Brennan, Stewart, Burgess et al., 2017; Davidson, 2010) and around $60 \%$ of patients regret their decision to commence dialysis (Davidson, 2010; Muthalagappan, Johansson, Kong et al., 2013), many reporting it was the wish of their physician or family. Locally, patients with renal failure are invited to a patient/carer education session providing detailed information about the different types of dialysis on offer, and individual sessions to revisit options and introduce conservative management. The group session lacked detail on conservative management and the renal supportive service provided by the local hospice.

Aim To improve the decision-making process ensuring patients fully understand all available options, reducing inappropriate commencement of dialysis and patient regret.

Method The author met with the renal nurse manager who provides dialysis modality education in the local area, and offered to deliver information on conservative management and the service provided by the local hospice.

Results To date there have been three collaborative education sessions, providing information to 17 patients and carers. Evaluation of these sessions are consistently positive. Comments include; 'informative, brilliant, clear explanation of options, we did not know there were so many options, thank you for the information and help'. At the latest session attended by two patients over the age of 75 with multiple comorbidities and their carers, both patients chose conservative management over dialysis.

Conclusion The success of this project demonstrated that inter-organisational collaboration between the hospice renal service and the hospital renal service enhanced the delivery of information, promoting fully informed patient-centred decision making. 